\title{
28 Research Square \\ Impact of Covid-19 on General Surgical Practice in India
}

\section{Amrit Manik Nasta ( $\square$ amritnasta@hotmail.com )}

Wockhardt Hospitals

\section{Ramen Goel}

Wockhardt Hospitals

Kanagavel Manickavasagam

St. Isabel's hospital

\section{Easwaramoorthy Sundaram}

Lotus Hospital, Erode

\section{Research Article}

Keywords: COVID-19, surgery, laparoscopy, safety, financial impact

Posted Date: June 25th, 2020

DOl: https://doi.org/10.21203/rs.3.rs-31151/v1

License: (c) (i) This work is licensed under a Creative Commons Attribution 4.0 International License. Read Full License 


\section{Abstract}

Introduction: The Coronavirus disease-2019 (COVID-19) pandemic is a global health crisis and surgeons are at increased occupational risk of contracting COVID-19. The impact of the disease on prevalent general surgical practice is uncertain and continues to evolve.

Aims/Objectives: To study the impact of COVID-19 on general surgical practice in India and the future implications of the pandemic.

Methods: A survey questionnaire was designed and electronically circulated one month after India entered a national lockdown during covid-19 pandemic, among members of Indian Association of Gastro-intestinal Endosurgeons (IAGES), a surgical association with nearly eight thousand members. Survey questions pertaining to preCOVID era surgical practices, impact on current practice and financial implications were asked. Responses were collected and statistically analyzed.

Results: 153 surgeons completed the survey, of which only $9.2 \%$ were females. $41 \%$ surgeons were more than 20 years into practice. $36.6 \%$ were into private practice at multiple hospitals (free-lancers). $41.8 \%$ had mainly laparoscopic practice with mean outpatient consultation of 26 patients/day and elective surgeries of 43 cases /month prior to lockdown. Post lock-down, daily outpatient consults reduced to 4 patients/day and $77 \%$ had not performed even a single elective procedure. $52 \%$ were taking hydroxychloroquine (HCQ) as chemoprophylaxis. $56.9 \%$ stated they are using personal protective equipment for all cases while $71.5 \%$ stated there are insufficient guidelines for future surgical practice in terms of safety. $52 \%$ surgeons stated a drop of more than $75 \%$ of their monthly income while $22 \%$ faced $50-75 \%$ reduction. $33 \%$ of respondents own a hospital and are expecting a monthly financial liability of 2.25 Million rupees (nearly 30,000 US dollars).

Conclusion: COVID 19 has led to a drastic reduction in outpatient and elective surgical practices. There is a definite need for guidelines regarding safety for future surgical practices and solutions to overcome the financial liabilities in the near future.

\section{Introduction}

The novel Corona virus was declared a public health emergency of international concern (PHEIC) by the World Health Organization (WHO) on 30 th January 2020[1]. In the first week on March, an unexpectedly high number of cases were detected worldwide and Corona Virus Disease 2019 (COVID-19) was declared a pandemic on $11^{\text {th }}$ March 2020[2].The Indian government announced a countrywide lockdown for three weeks starting at midnight on 24 March to slow the spread of covid-19 as the number of people testing positive in the country reached 563[3]. However, this lockdown was further extended till $3^{\text {rd }}$ May 2020. One month into lockdown, outpatient clinics and elective surgeries were likely to have taken a beating. Majority of hospital resources were directed towards availing masks and personal protective equipment (PPE), minimizing staff movements and suspension of all elective work.

\section{Aims}

To study the impact of COVID-19 on general surgical practice in India and the future implications of the pandemic. 


\section{Methods}

This survey was conducted at a tertiary-care hospital. The survey questionnaire was designed and electronically circulated one month after India entered a national lockdown, among members of Indian Association of Gastrointestinal Endo-surgeons (IAGES), nearly 8000 member strong association with surgeons having interest in general and laparoscopic surgery.

Survey questions pertaining to pre-COVID era surgical practices, impact on current practice and financial implications were asked. Responses were collected and Chi square test was used for statistical analysis.

The self-administered questionnaire consisted of twenty one questions with five part socio- demographic questions, questions on outpatient and surgical (emergency/elective) numbers in pre and post-lockdown period, safety practices and financial impact in the current period.

\section{Results}

One hundred and fifty three surgeons from across the country completed the survey, of which only $9.2 \%$ were women. Amongst the respondents, $41.2 \%$ surgeons were more than 20 years into practice, $34 \%$ for 10 to 20 years and $22.2 \% 4$ to 10 years after completing their specialty degree. For place of practice, $36.6 \%$ were into private practice at multiple hospitals (free-lancers), $29.4 \%$ were full timers at a single corporate hospital and $13.1 \%$ were full timers at government hospitals.

Prior to lockdown: Amongst the respondents, $41 \%$ had mainly laparoscopic practice and $41.7 \%$ had equal proportion of laparoscopic and open surgery (Figure 1). Surgeons reported a mean outpatient consultation of 26 patients/day and elective surgeries 42 cases/month prior to lockdown.

Post lockdown: Since the beginning of lockdown, 36.5\% reported to have completely stopped outpatient services, $63.5 \%$ surgeons had a reduction in their services and $50 \%$ reported to have started online consultations. Amongst those continuing consultations, average daily consults reduced to 4 patients per day.

All elective surgical work was stopped by $93.3 \%$ while $5.2 \%$ had scaled down elective surgeries. No elective procedures were performed by $77 \%$, while $16 \%$ performed less than 5 surgeries during the lockdown period. No emergency surgeries were performed by $9 \%$ surgeons, and $42.5 \%$ had reduced emergency services where feasible. Average elective and emergency surgeries performed in the month of lockdown were merely one and five in number respectively (Figure 2). The reduction in OPD, elective and emergency surgical practice was statistically significant $(p<0.05)$.

Safety practices: Hydroxychloroquine (HCQ) was taken by $52 \%$ surgeons for chemoprophylaxis. It was felt by $93 \%$ surgeons that laparoscopic surgery and use of energy sources increased the risk of aerosol spread of the virus. For safe surgical practice, $56.9 \%$ of surgeons said they would use PPE in all cases, $38.5 \%$ would prefer open surgery and $33.1 \%$ would use filters for de-sufflation. More evidence was sought by $71.5 \%$ of surgeons to understand safety practices in future (Figure 3).

Half the surgeons (50.8\%) claimed COVID-19 rapid antigen test had false negative rate of up to $30 \%$, while $43.1 \%$ claimed high false negative antibody rates are seen in first week of infection. When asked to select top three areas where adequate information is lacking on COVID-19 for surgeons, most surgeons selected future of surgery in COVID era (77.7\%), safety in laparoscopy (69.2\%) and safety of staff (59.2\%) (Figure 4). For sources of COVID-19 
information, 76.1\% utilized internet as a medium, 5.9\% utilized Television, while $15.4 \%$ utilized directives given on government/national body sources.

Financial impact:

A drop of more than $75 \%$ of their monthly income was experienced by $52 \%$ surgeons, while $22 \%$ faced $50-75 \%$ reduction. Subgroup analysis revealed surgeons working in private hospitals had significantly $(P=0.000)$ greater reduction in income, compared to surgeon in government set up (Figure 5). One third (33\%) of respondents owned a hospital and were expecting a monthly financial liability of 2.25 Million rupees (30,000 US dollars).

\section{Discussion}

COVID-19 has led to significant worldwide change in surgical practice. Our survey showed that practice of majority of surgeons in India has been drastically affected by the COVID-19 pandemic. As of $24^{\text {th }}$ April, 2020 at 9 am, over 23,000 cases were confirmed positive cases in India [4].

Outpatient consultations and non-emergency surgeries have declined from over 20 cases/day and 40 surgeries/month to almost zero cases in this lockdown period. Spinelli [5] reported that most outpatient clinics were suspended in Italy, and scheduled patients are called beforehand by hospital administration, asking for specific symptoms in the previous two weeks (for example, fever or cough), or direct exposure to COVID19-positive individuals. In such cases, the patient was asked not to come to the hospital and the visit was postponed. A survey on ophthalmology practice in India by Nair et al. [6] showed the $72.5 \%$ of the practicing ophthalmologists in India were in total lockdown. While the clinicians themselves may be available, the unavailability of managerial, administrative, nursing and other support staff may pose logistical and operational challenges in running a health care facility during covid-19 pandemic.

Surgeons are at dual risk of exposure in their practice namely both from the outpatient clinics and operating room. Our survey showed that nearly $50 \%$ surgeons have taken hydroxychloroquin (HCQ) as chemoprophylaxis. A systematic review by Shah et al. [7] showed an absence of robust in vivo evidence to support the role of HCQ in prevention of COVID-19. But the National Task Force for COVID-19 constituted by Indian Council of Medical Research (ICMR) on March 22, 2020 recommended HCQ for prophylactic use in asymptomatic healthcare workers (HCWs) involved in care of suspected or confirmed patients of COVID-19 [8]. When HCQ administration is considered for a COVID-19 patient or suspect, efforts should be made to advise high-risk individuals to have a baseline ECG recording [9].

After the pandemic is under control, it is unclear on the extent of precautions that would be required to be followed by surgical and operating room personnel. When asked about future concerns, most surgeons mentioned regarding the future of surgical practice in COVID era (78\%), safety in laparoscopy (69\%) and safety of staff (59\%). This mandates the need for national and international guidelines for addressing these issues. Stahel [10] has provided an algorithm based on elective surgical indications and predicted proper perioperative utilization of critical resources, including the consideration for intra/postoperative blood product transfusions, estimated postoperative hospital length of stay, and the expected requirement for prolonged ventilation and need for postoperative ICU admission.

Prior to COVID-19 lockdown in India, 42.2\% surgeons in our survey mainly did laparoscopic practice. According to a study by Tuech et al. [11], caution is required when performing laparoscopy because of the risk of aerosol release 
and subsequent risk of exposure to operating room personnel. The main risk comes from the possible presence of pathogens in the peritoneal cavity. The aerosol released into the theatre during surgery from ports or after the operation (de-sufflation of abdomen), may contaminate personnel and equipment and surfaces in the room via airborne particles [11]. In our survey, 56.9\% claimed they would use PPE in all cases, 38.5\% would prefer open surgery and $33.1 \%$ would use filters for de-sufflation while $71.5 \%$ stated more evidence was required. Despite guidelines coming from various national and international surgical societies, there is still a major uncertainty about what safe practices are to be adopted.

Our survey reported that $77 \%$ of surgeons relied on internet as a premium source of COVID-19 information. Currently, the vast diversity of information available through the Internet, including unverified malicious information, can spread quickly and can misguide Healthcare workers (HCWs). Health authorities and scientists have warned that widespread misinformation about COVID-19 is a serious concern causing xenophobia worldwide [12]. Another concern on surgical practice is the economic impact of the pandemic. Our survey reported $52 \%$ surgeons experienced a $75 \%$ or more reduction in their income, and the duration of this scenario is uncertain. In a recent survey conducted by Irish Dental Association (IDA) which involved 369 dentists, it was reported that one-fifth of the dentists have closed their practices (temporarily or permanently). In addition, around three-fourth of the participants are expecting a financial loss of over 70\% amid COVID-19 outbreak [13].

A limitation of the study was that COVID has affected different cities and states in India with variable intensity hence the perceptions of the responders will be limited.

\section{Conclusion}

Our survey highlights the need for more information on future of surgical practices, to make surgery safe in the times of the pandemic. The existing lockdown has had a major impact on routine surgical practice and will require dedicated efforts for resumption of "new normal" in future of laparoscopic surgery.

\section{Declarations}

The authors declare no conflict of interest. Need for informed consent was waived due to retrospective nature of study after approval from Institutional Review Board.

\section{References}

1. Eurosurveillance Editorial Team. Note from the editors: World Health Organization declares novel coronavirus (2019-nCoV) sixth public health emergency of international concern. Euro Surveill. 2020 Feb;25(5):200131e.

2. WHO Director-General's opening remarks at the media briefing on COVID-19 - 11 March 2020. (2020). Accessed: Mar 19, 2020: https://www.who.int/dg/speeches/detail/who-director-general-s-opening-remarks-at-the-mediabriefing-on-covid-19-11-March-2020.

3. Pulla P. Covid-19: India imposes lockdown for 21 days and cases rise. BMJ 2020;368:m1251

4. ICMR testing update

$24^{\text {th }}$ April, https://icmr.nic.in/sites/default/files/whats_new/ICMR_testing_update_24Apr2020_9AM_IST.pdf

5. Spinelli A, Pellino G. COVID-19 pandemic: perspectives on an unfolding crisis. Published online in Wiley Online Library (www.bjs.co.uk). DOI: 1002/bjs.11627

Page 5/10 
6. Nair AG, Gandhi RA, Natarajan S. Effect of COVID-19 related lockdown on ophthalmic practice and patient care in India: Results of a survey. Indian J Ophthalmol2020;68:725- 30.

7. Shah S, Sad S, Jain A, Misra DP, Negi VS. A systematic review of the prophylactic role of chloroquine and hydroxychloroquine in Coronavirus Disease-19 (COVID-19). Internation Journal of Rheumatic Diseases. April 2020. doi:10.1111/1756-185X.13842

8. https://www.mohfw.gov.in/pdf/AdvisoryontheuseofHydroxychloroquinasprophylaxisforSARSCoV2infection.pdf

9. Aditya Kapoor, Ulhas Pandurangi, Vanita Arora, Anoop Gupta, Aparna Jaswal, Ashish Nabar, Ajay Naik, Nitish Naik, Narayanan Namboodiri, Amit Vora, Rakesh Yadav, Anil Saxena. Cardiovascular risks of hydroxychloroquine in treatment and prophylaxis of COVID-19 patients: A scientific statement from the Indian Heart Rhythm Society. Indian Pacing and Electrophysiology Journal, 2020, ISSN 09726292.https://doi.org/10.1016/j.ipej.2020.04.003.

10. Stahel P. How to risk-stratify elective surgery during the COVID-19 pandemic? Patient Safety in Surgery (2020) 14:8 https://doi.org/10.1186/s13037-020-00235-9

11. Tuech J, Gangloff A, Di Fiore F, Michel P., Brigand C, Slime K., Pocard M., Schwarz L. Strategy for the practice of digestive and oncological surgery during the Covid-19 epidemic. Journal of Visceral March 2020. https://doi.org/10.1016/j.jviscsurg.2020.03.008

12. Lai CC, Shih TP, Ko WC, Tang HJ, Hsueh PR. Severe acute respiratory syndrome coronavirus 2 (SARS-CoV-2) and corona virus disease-2019 (COVID-19): the epidemic and the challenges. Int J Antimicrob Agent. 2020:105924. Doi:10.1016/j.jjantimicag.2020.105924.

13. IDA Scale of dental collapse highlighted in survey. Available: http://irishdentalassociation.newsweaver.com/newsletter/13ctcsav7cz1cys22xtzj2?a=1 $\& p=56657247 \& t=22322575$

\section{Figures}




\section{Types of Surgical practice}

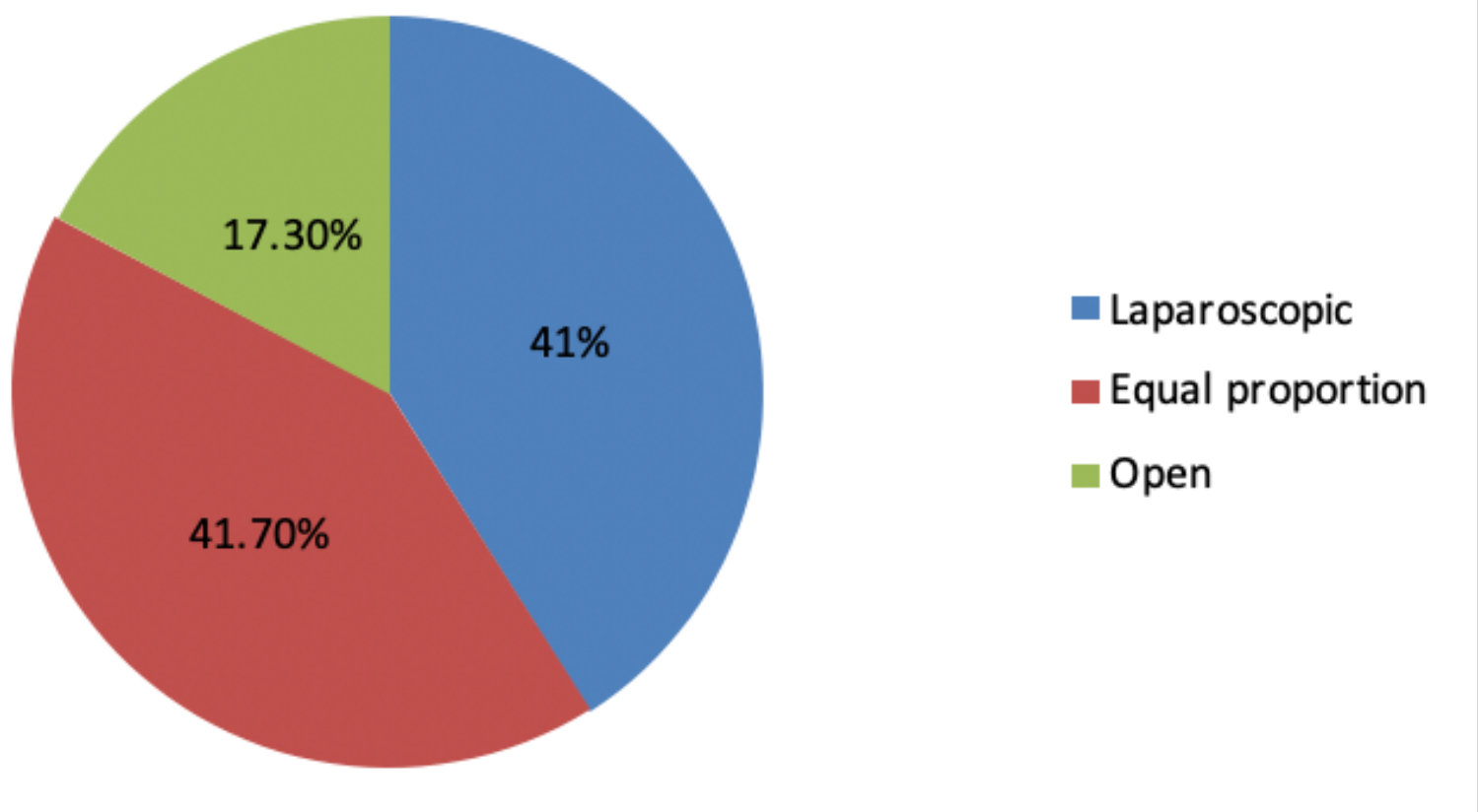

Figure 1

Distribution of open and laparoscopic surgical practice prior to lockdown

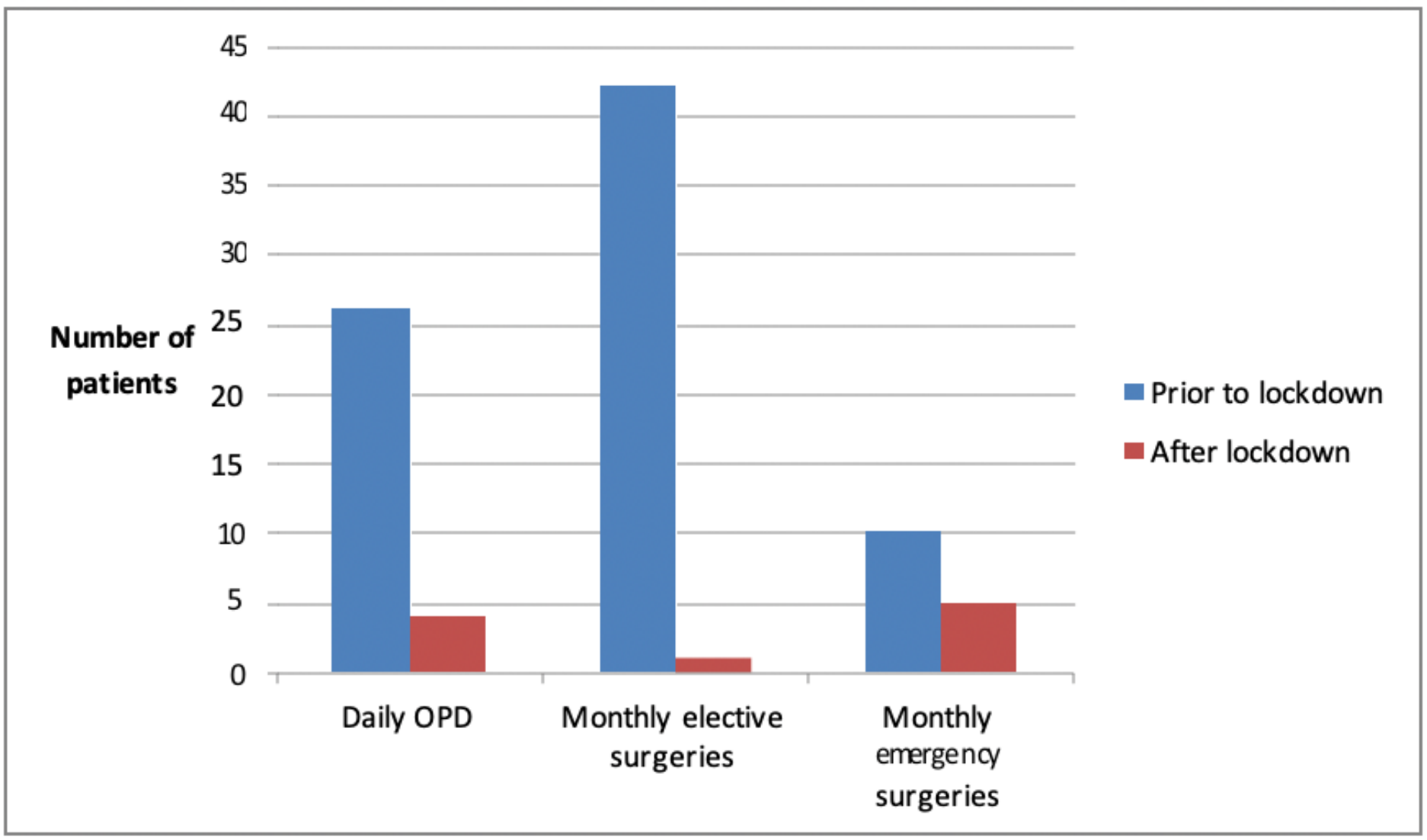


Figure 2

Comparison of daily Outpatient consultations (OPD), monthly elective and emergency surgeries prior and postlockdown

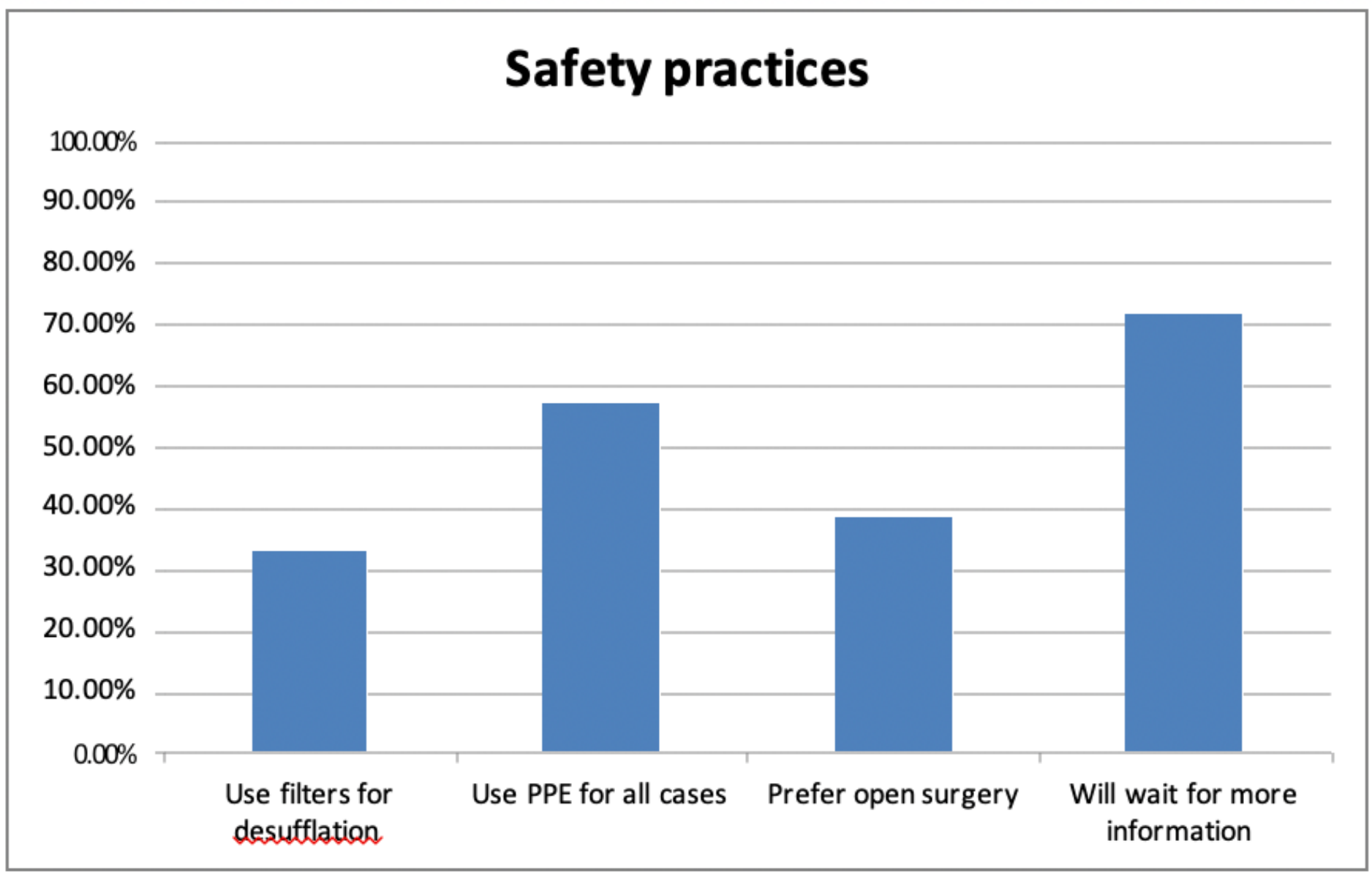

Figure 3

Safety measures to be adopted for future surgeries 


\section{Top 3 areas where information is lacking}

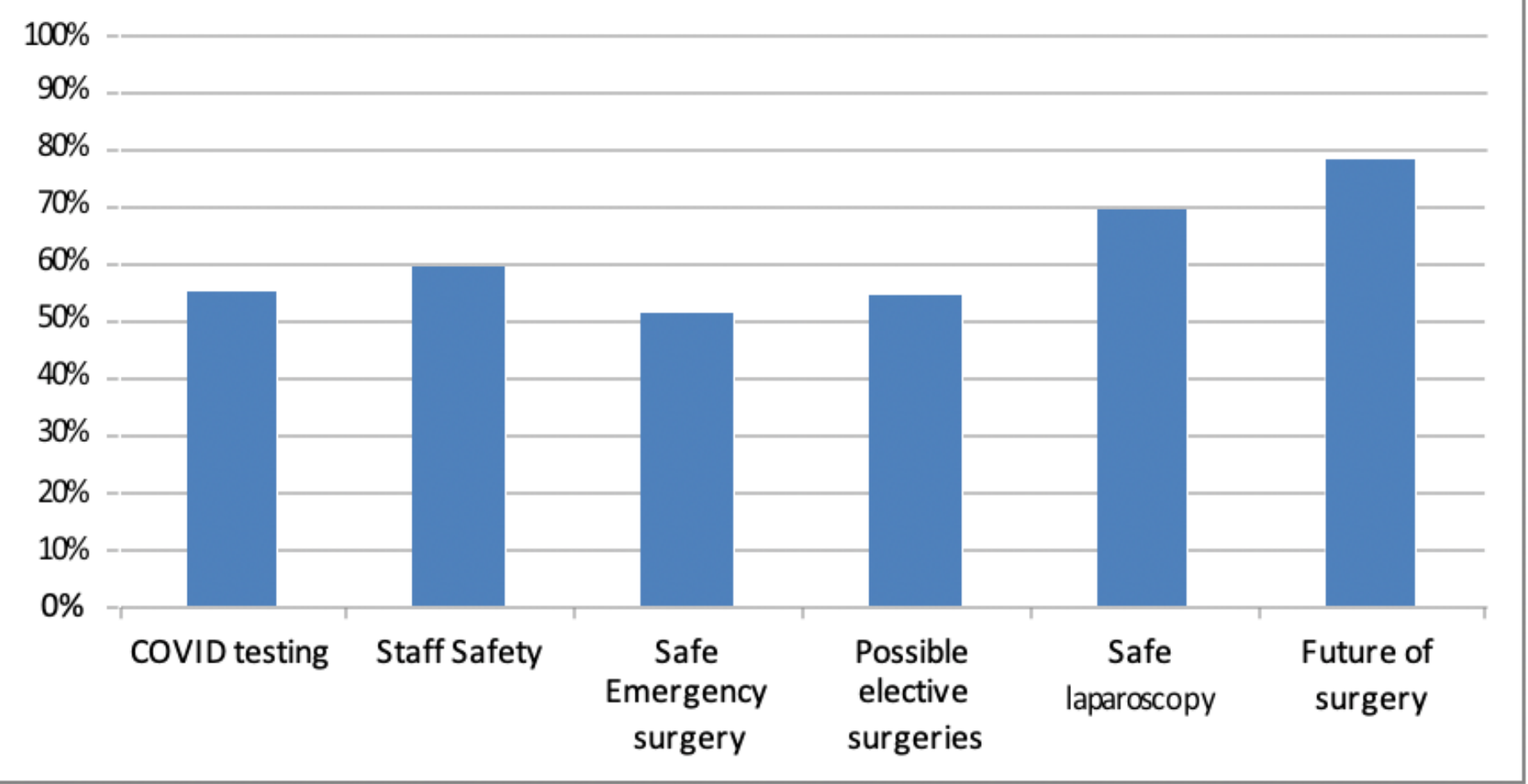

Figure 4

Top three areas where information is lacking for surgery 


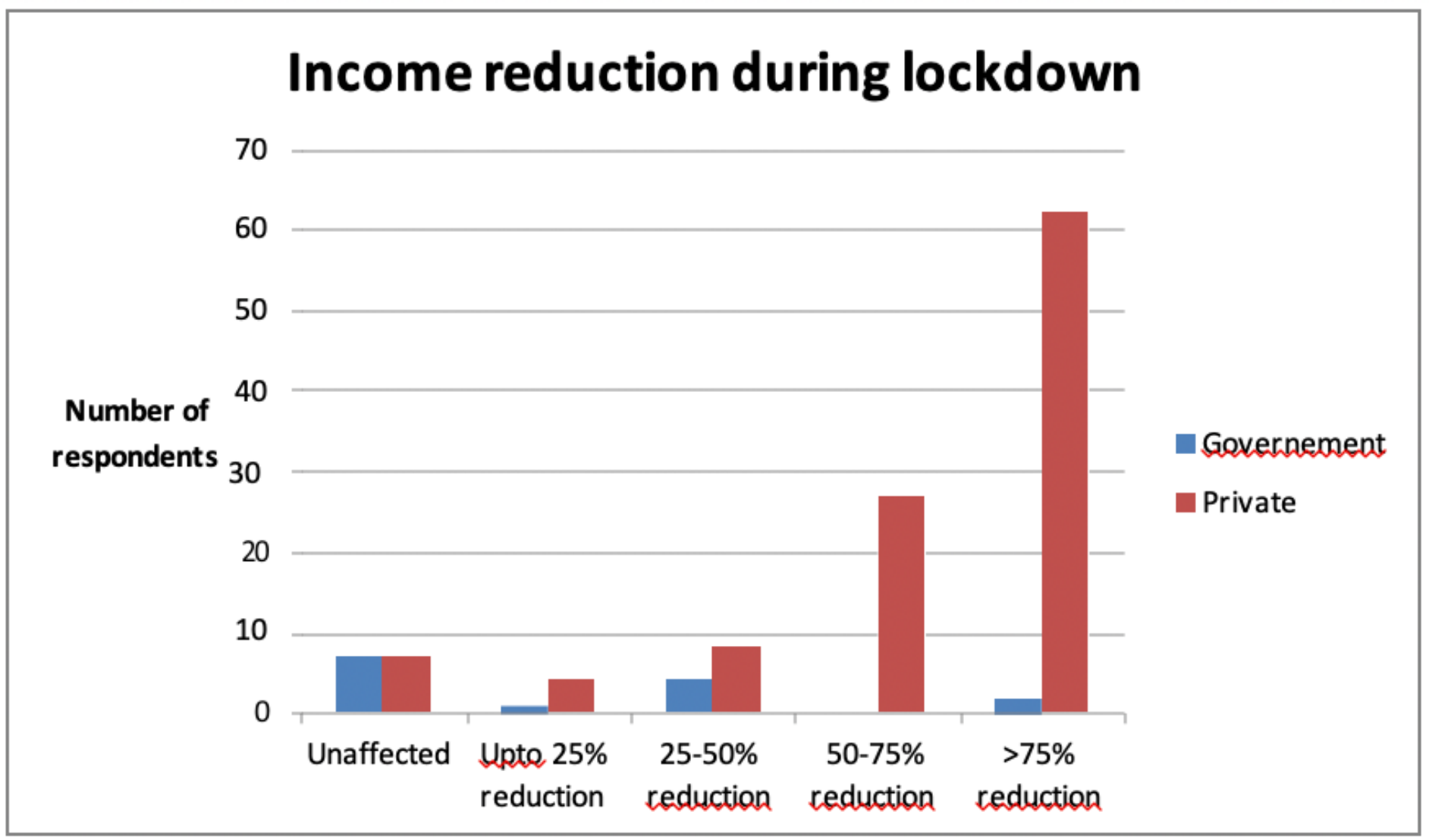

Figure 5

Comparison of financial losses in surgeons working in private versus government setup 